Research article

\title{
Irradiation provided by dental radiological procedures in a pediatric population
}

\author{
Mihaela Hedesiu $^{\mathrm{a}}$, Maria Marcu ${ }^{\mathrm{a}, *}$, Benjamin Salmon $^{\mathrm{b}}$, Ruben Pauwels ${ }^{\mathrm{c}, \mathrm{d}}$, \\ Anne Caroline Oenning e, Oana Almasan ${ }^{\mathrm{a}}$, Raluca Roman ${ }^{\mathrm{a}}$, Mihaela Baciut ${ }^{\mathrm{a}}$, Reinhilde Jacobs ${ }^{\mathrm{c}, \mathrm{f}}$, \\ DIMITRA Research Group ${ }^{1}$
}

a Department of Oral and Maxillofacial Radiology, Faculty of Dentistry, "Iuliu Hatieganu" University of Medicine and Pharmacy, Cluj-Napoca, Romania

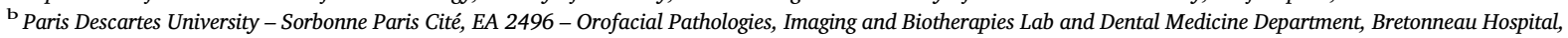
HUPNVS, AP-HP, Paris, France

c OMFS-IMPATH Research Group, Department of Imaging and Pathology, Faculty of Medicine, Catholic University of Leuven, and Oral \& Maxillofacial Surgery, University Hospitals Leuven, Leuven, Belgium

d Department of Radiology, Faculty of Dentistry, Chulalongkorn University, Bangkok, Thailand

e Department of Oral Diagnosis, Division of Oral Radiology, Piracicaba Dental School, University of Campinas (UNICAMP), Piracicaba, Sao Paulo, Brazil

${ }^{\mathrm{f}}$ Department of Dental Medicine, Karolinska Institutet, Stockholm, Sweden

\section{A R T I C L E I N F O}

\section{Keywords:}

Radiation dose

Radiation risk

Pediatric radiology

CBCT

Radiosensitivity

\begin{abstract}
A B S T R A C T
Background: Children are more sensitive to ionizing radiation effects due to their high radiosensitivity. Purpose: To estimate doses and risks for dental radiological examinations in children.

Material and methods: A pediatric population consisting of 7150 children and young adults which underwent 12252 dental radiological examinations (4220 intraoral, 1324 cephalometric, 5284 panoramic radiographs and 1424 CBCTs) within two years were included. Two groups were studied: CBCT group (exposed to $\mathrm{CBCT} \pm$ conventional radiographs) and 2D group (exposed only to 2D radiological examinations). The effective doses were corrected according to age at exposure and settings parameters (mA;FOV) by using logarithmic fit equations for dose interpolation. The individual cumulative dose, per-caput collective dose and radiation risk were calculated for each group.

Results: The median effective and cumulative doses for conventional radiographs were lower than $20 \mu S v$ and did not vary with age. Children exposed to CBCT had a higher median effective dose (127.2 $\mu$ Sv) and cumulative dose $(156.5 \mu \mathrm{Sv})$ with a significant increased cumulative dose between 11 and 14 years. The CBCT contributed with $70 \%$ to the collective dose and per caput collective dose was $184 \mu \mathrm{Sv}$ for CBCT exposures. The Life Attributable Risk (LAR) and Relative Radiation Level (RRL) were significantly higher for children exposed to CBCT under the age of 18. The highest radiation dose for CBCT was equivalent with 34.1 days of natural background radiation and it was found for ages between 11 and 15 .

Conclusion: The CBCT doses and radiation risk vary but remain in the lower levels of the relative risk of medical exposures.
\end{abstract}

\section{Introduction}

Although dental radiological examinations deliver a low dose of radiation, they represent one third of all radiological examinations in Europe [1]. The use of CBCT in children population has recently increased and a particular attention regarding radiation protection is needed [2].

The SedentexCT guidelines showed that CBCT examinations should be recommended to clinical situations in which the information provided may change the diagnosis or improve the treatment plan [3]. However, the efficacy of CBCT in revealing the three-dimensional morphology of maxillofacial bone structure has led to increased examinations in many fields of dentistry, including orthodontics [4].

The American Academy of Oral and Maxillofacial Radiology (AAOMR) has revealed the efficacy of CBCT for dental anomalies and treatment planning in moderate and severe skeletal discrepancies and

\footnotetext{
*Corresponding author at: Department of Oral and Maxillofacial Radiology, Faculty of Dentistry, University of Medicine and Pharmacy "Iuliu Hatieganu" Cluj-Napoca, 400012, Romania.

E-mail address: marcumaria88@gmail.com (M. Marcu).

${ }^{1}$ List of partners on www.dimitra.be.
} 
Table 1

The effective doses and dosimetric methods reported in the literature for CBCT and 2D examinations on radiological units used in the current study.

\begin{tabular}{|c|c|c|c|c|c|c|c|}
\hline X-ray unit & Dosimetric method & Phantom & $\mathrm{kV}$ & mAs & $\begin{array}{l}\text { Scanning volume }(\mathrm{WxH}) \\
\left(\mathrm{cm}^{2}\right)\end{array}$ & $\begin{array}{l}\text { Effective dose } \\
(\mu \mathrm{Sv})\end{array}$ & References \\
\hline \multirow[t]{4}{*}{ NewTom 3G (Cefla Dental Group, Imola, Italy) } & OSLD & 10-years-old & 110 & 9 & $15 \times 15$ & 94 & Ludlow et al. [11] \\
\hline & & child & 110 & 9 & $20 \times 20$ & 56 & Ludlow et al. [11] \\
\hline & & adult & 110 & 9.1 & $15 \times 15$ & 57 & Loubele 2009 [15] \\
\hline & & & 110 & 9 & $30 \times 30$ & 30 & Loubele 2009 [15] \\
\hline \multirow[t]{9}{*}{ ProMax 3D Max (Planmeca, Finland) } & TLD & 10-years-old & 84 & 19.6 & $8 \times 8$ & 24 & Theodorakou [16] \\
\hline & & child & 84 & 19.9 & $8 \times 8$ & 28 & Pauwels et al. [6] \\
\hline & & & 90 & 217 & $16 \times 16$ & 277 & Ludlow et al. [11] \\
\hline & & adult & 84 & 192 & $8 \times 5^{a}$ & 171 & Qu et al. [17] \\
\hline & & & 84 & 192 & $8 \times 5^{b}$ & 131 & Qu et al. [17] \\
\hline & & & 84 & 169 & $8 \times 8$ & 122 & Pauwels et al. [6] \\
\hline & & & 84 & 168 & $8 \times 8$ & 272 & Qu et al. [17] \\
\hline & & & 90 & 325 & $16 \times 16$ & 283 & Ludlow et al. [18] \\
\hline & & & 90 & 271 & $16 \times 16$ & 223 & Ludlow et al. [18] \\
\hline \multirow[t]{3}{*}{ Scanora 3D (Soredex, Tuusula, Finland) } & TLD & adult & 85 & 48 & $14.5 \times 13.5$ & 68 & Pauwels et al. [6] \\
\hline & & & 85 & 30 & $10 \times 7.5^{\mathrm{b}}$ & 46 & Pauwels et al. [6] \\
\hline & & & 85 & 30 & $10 \times 7.5^{\mathrm{a}}$ & 47 & Pauwels et al. [6] \\
\hline CranexTome(CCD) (Soredex, Finland) & TLD & adult & 70 & 64 & Panoramic & 8.1 & Gijbels et al. [19] \\
\hline ProMax 3D (SPP) (Planmeca, Finland) & TLD & adult & 66 & 144 & Panoramic & 8 & Al-Okshi et al. [20] \\
\hline \multirow{3}{*}{$\begin{array}{l}\text { Intraoral, indirect digital exposure, round } \\
\text { collimation }\end{array}$} & TLD & & 70 & 25.6 & Bitewing & 0.6 & Ludlow et al. [11] \\
\hline & & & & & Periapical & 1.9 & \\
\hline & & & & & Occlusal & 7 & \\
\hline
\end{tabular}

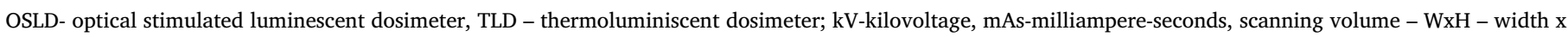
height, $\mu \mathrm{Sv}$-microsievert, a - for mandibular exposure, b- for maxillary exposure

the median age of children exposed to CBCT was found to be 12 years [5].

A wide range in patient dose for dental СВCT has been reported in literature [6]. CBCT was described as a low-dose radiological method but in fact it can reach doses similar to those of medical CT in using large field of view (FOV) and high-resolution protocols [7]. In light of the need to weigh the benefits and the risks of radiological exposure from a pediatric perspective, the DIMITRA project (Dentomaxillofacial pediatric imaging: An Investigation Towards Low Dose Radiation Induced Risks) aims to characterize the doses and the potential biological effects of radiological exposures in pediatric dentistry [8].

The estimation of doses and a potential radiation risk in children through epidemiological studies could increase the efficacy of using the patient - oriented CBCT protocols in dentistry. However, the evaluation of СBCT doses under clinical condition is still a difficult issue due to the variability of the scanning protocols and CBCT units [9]. The majority of CBCT doses currently estimated in literature involved dosimetric measurements on anthropomorphic phantoms. However, a large discrepancy was observed between phantoms and patient doses, mainly for children, due to immature tissues, growth potential and their developmental stage [10].

In addition, even the dosimetric measurements revealed higher effective doses for СВСТ compared to other dental radiological investigations [11] the question that arises is whether CBCT significantly influenced the radiation risk considering the age and frequency in using CBCT for children.

Several studies demonstrated that the risk of cancer incidence may theoretically be increased after repeated radiological procedures during childhood and adolescence for various medical exposures such as interventional radiology [12], multiple CT for emergency situations [13] or cardiac imaging radiology [14]. However, until now there has been no evidence of a potential oncologic effect of low dose radiological examinations. Moreover, the extrapolation of the linear no-threshold model (LNT) that provides the estimated cancer risk for high dose exposures is very controversial and it is considered of little relevance for doses less than $100 \mathrm{mSv}$.

Regardless of technical difficulties in estimating the CBCT effective doses in clinical condition, an overall estimation of the CBCT contribution in dental radiological exposures for various ages of children still remains of great interest.

The aim of this study was to estimate the overall differences between a group of patients exposed to CBCT compared with a group exposed only with $2 \mathrm{D}$ dental radiographic modalities, with regard to the variation of the cumulative dose and radiation risks in a pediatric population.

\section{Materials and methods}

\subsection{Patient inclusion and data collection}

A retrospective cohort study was conducted in five oral radiology departments from two European countries (Romania and France), with a long lasting expertise in oral and facial radiology. The selected population included children and young adults, aged between 0 and 22 years, who underwent at least one dental radiological examination (CBCT or 2D dental X-ray) within two years (from 1st January 2014 to 31st December 2015).

The selected population included healthy children who underwent a radiography for screening diagnosis of dental lesions (e.g. bite-wing radiography for caries detection) and also children with various dental or maxillofacial pathologies. The following data regarding exposure was collected from the radiological units: personal data (age and gender), radiological examination (type of examination and equipment) and exposure protocol (FOV, $\mathrm{kV}, \mathrm{mAs}$ ). Only patients with a complete set of information regarding the exposure were included in the study. The lack of clinical data for which the patients were referred to dental radiological examination was not considered as an exclusion criterion in the present study.

The pediatric population was divided into two groups: children who underwent a CBCT $\pm 2 \mathrm{D}$ dental radiological examination within two years (CBCT group) and children who underwent only 2D dental radiological examination (2D group). Each group was also classified according to age at the time of the radiological exposure.

The ethics committee of "Iuliu Hatieganu" University of Medicine and Pharmacy Cluj-Napoca, Romania has approved the epidemiological study (230/5.05/2015) and ethical committee of Katholieke Universiteit Leuven, Belgium has approved the DIMITRA project (S5694/15.07.2015). 


\subsection{Estimation of radiation doses}

The x-ray units used for the CBCT and 2D dental radiological procedures in the selected centers are presented in Table 1. For this type of units, the most suitable effective doses (ED) reported in the literature were considered according to the type of radiological examination and the exposure protocol used for the pediatric population [6,11,15-20]. The effective doses presented in Table 1 were calculated on child and adult anthropomorphic dosimetric phantoms and were considered for the estimation of radiation doses in children. The quality control tests for radiographic and CBCT exposures have shown that all radiological equipments operated at their technical performance level in all included radiological centers within the time period of the study.

The CBCT effective doses reported in literature were then corrected according to $\mathrm{mAs}$ and FOV used for the radiological exposure of the selected population. A previously published volume-dose model for $\mathrm{CBCT}$, in which the $\mathrm{ED} / \mathrm{mAs}$ was expressed as a function of FOV diameter and height based on a set of measurements obtained using various FOVs, was used [9]. A CBCT-dependent correction factor for ED/ mAs was obtained by using at least two values of the effective doses measured on anthropomorphic phantoms with different FOVs and the logarithmic fit equation of the volume parameter (diameter $\times$ height ${ }^{2}$ ). The logarithmic fit equations for FOV dose correction are presented in Appendix (A) in Supplementary material. The effective doses for CBCT exposures were then calculated based on the tube current exposure time used in clinical condition for the selected pediatric population.

The coefficient for the adjustment of CBCT doses to the age of the pediatric cohort was obtained by using the linear interpolation of the $\mathrm{ED} / \mathrm{mAs}$ parameter estimated in 10- years-old child phantom and adult phantoms using the ProMax 3D CBCT unit, with a large FOV scan $(16 \times 16 \mathrm{~cm})$ and $90 \mathrm{kV}$ exposure protocol. All equations for linear extrapolation of doses according to age and the logarithmic fit equations for FOV are presented in the Appendix (A) in Supplementary material.

The effective doses for conventional radiological exposures in the pediatric population were corrected for tube current exposure time and a linear interpolation with age was also applied. The $\mathrm{kV}$ was fixed in $92 \%$ of cases examined by CBCT and no correction of doses was applied for $\mathrm{kV}$ in the current study.

The total individual cumulative dose for dental radiological exposures represents the total amount of radiation given to a patient considering one-year lag for multiple exposures. The individual cumulative dose for the CBCT group of patients was calculated for all dental exposures including CBCT and conventional x-rays. The median $\left(\mathrm{Q}_{2}\right)$, interquartile range (IQR) and the maximum value of the cumulative and effective dose were calculated at different ages for the CBCT group and 2D group of patients (Table 2).

For the CBCT group, the cumulative dose incurred by each person according to dental radiological procedures was calculated as follows: the cumulative dose for a single CBCT (single CBCT dose), for repeated CBCTs (multiple CBCTs dose) and for conventional dental x-rays (2D_CBCT group) (Fig. 1).

The collective effective dose for the selected population represents the total radiation dose incurred by the selected population from dental radiological exposures. Per caput collective dose was estimated dividing the collective doses by the number of patients included in the study (Table 3).

\subsection{Estimation of radiation risk}

The radiation risk was estimated using three different methods: the relative radiation level (RRL), estimation of the lifetime attributable risk for cancer incidence (LAR), the background equivalent radiation time (BERT) (Tables 2 and 3 respectively).

The individual LAR for cancer incidence was estimated for various ages of males and females using the BEIR VII preferred model
Table 12D-1 [21] and represents the number of cancer cases per 100,000 persons which were exposed to a single dose of $0.1 \mathrm{~Gy}$. The individual LAR for each child was calculated using linear interpolation of risk inside of the age interval and the effective dose for each exposure. The radiation risk was expressed only according to dental radiological examinations and the total individual risk for multiple exposures represents the sum of risks for each exposure.

Background equivalent radiation time (BERT) represents the amount of radiation received from a radiological procedure expressed in terms of number of days of background radiation [22]. It was calculated for the studied groups considering per caput dose for a certain radiological examination and a background radiation of $3 \mathrm{mSv} /$ year (Table 3).

RRL was used to compare the amount of radiation given by the radiological examinations with a variable effective dose as follow:( $(*)$ radiation dose $<30 \mu \mathrm{Sv}$ for ages under 18 or radiation dose $<100 \mu \mathrm{Sv}$ for ages over 18 years; $(\because)$ ) radiation dose between 30 and $300 \mu \mathrm{Sv}$ for ages under 18 years [23].

\subsection{Data analysis and statistics}

In order to calculate the individual cumulative dose of dental radiological examinations, LabVIEW Professional for Windows v.2017 (NI, Austin, Texas, USA) was used for merging the effective doses of the radiological procedures based on the patient's name and date of birth. Median value, interquartile range and maximum values were calculated for the effective and cumulative doses. Statistical analysis of differences between CBCT group and 2D group of patients, was performed using Mann Whitney $U$ test for the non-normally distributed data and p-values $<0.05$ were considered statistically significant.

\section{Results}

\subsection{Descriptive epidemiology}

The cohort included 7150 children and young adults aged between 0 and 22 years. A total number of 12252 dental radiological examinations (4220 intraoral, 1324 cephalometric, 5284 panoramic radiographs and 1424 CBCTs) were performed on the selected pediatric population. Table 2 shows a summary of the distribution of cases according to age at exposure and studied group, the variation of the effective dose for the CBCT and conventional radiological examinations as well as the total individual cumulative doses for each group.

The CBCT group included 1009 children with a single CBCT exposure $(71.4 \%)$ or multiple CBCTs $(28,5 \%)$ from which only $2 \%$ underwent also a 2D radiological examination within the two years interval.

\subsection{Radiation doses for the pediatric cohort}

The median effective doses for 2D dental radiological exposures were lower than $20 \mu \mathrm{Sv}$ which represents the lowest range of RRL ( $\because$ ) while a higher level of radiation $(\because *)$ was found for the CBCT examinations perform at ages under 18 (Table 2).

The individual cumulative dose in the CBCT group was found to be significantly higher for children aged between 11 and 14 years old than in other age groups $(p<0.0001)$. The cumulative dose for the repeated CBCTs was significantly higher than for a single CBCT (median is $121.2 \pm$ IQR $118.2 \mu \mathrm{Sv}$ and $309.4 \pm$ IQR $222.1 \mu \mathrm{Sv}$ respectively) $(\mathrm{p}<0.05)$ and it exceed $1 \mathrm{mSv}$ in children with multiple CBCT exposures. There are no significant differences between the 2D and CBCT group in terms of the cumulative doses incurred by patients from $2 \mathrm{D}$ dental X-rays (Fig. 1).

Per caput collective dose was found to be increased for children exposed to CBCT compared to 2D group and it was statistically significantly higher for children exposed to CBCT at the ages between 11 
Table 2

Distribution of the irradiated patients, effective doses and individual cumulative doses according to age and studied groups.

\begin{tabular}{|c|c|c|c|c|c|c|c|c|c|c|c|c|}
\hline \multirow[t]{3}{*}{ Age group (years) } & \multicolumn{6}{|c|}{ Patients non-exposed to CBCT (2D group) } & \multicolumn{6}{|c|}{ Patients exposed to CBCT (CBCT group) } \\
\hline & \multirow[t]{2}{*}{$\mathrm{N}$} & \multicolumn{2}{|c|}{ 2D Effective Dose ( $\mu$ Sv) } & \multicolumn{3}{|c|}{ Cumulative Dose ( $\mu$ Sv) } & \multirow[t]{2}{*}{$\mathrm{N}$} & \multicolumn{2}{|c|}{ CBCT Effective Dose ( $\mu \mathrm{Sv})$} & \multicolumn{3}{|c|}{ Cumulative dose ( $\mu$ Sv) } \\
\hline & & $\mathrm{Q}_{2}$ & IQR & $\mathrm{Q}_{2}$ & IQR & Max & & $\mathrm{Q}_{2}$ & IQR & $\mathrm{Q}_{2}$ & IQR & $\operatorname{Max}$ \\
\hline $0-6$ & 114 & 7.6: & 10.1 & 11.4 & 14.5 & 114.9 & 26 & $51.5 \div$ & 97.6 & 67.4 & 111.3 & 336.7 \\
\hline $7-8$ & 315 & 5.8 & 10.5 & 11.4 & 13.9 & 92.1 & 44 & $137.6 \div$ & 90.2 & 135.1 & 102.7 & 433.9 \\
\hline $9-10$ & 534 & $5.4 \%$ & 10.5 & 11.4 & 16.4 & 182.3 & 91 & 132.2: & 49.1 & 136.7 & 44.9 & 722.0 \\
\hline $11-12$ & 522 & $3.2:$ & 9.9 & 11.2 & 17.3 & 105.5 & 155 & 204.8: & 66.5 & $214.9^{*}$ & 145.7 & 1251 \\
\hline $13-14$ & 543 & $2.6 \%$ & 9.6 & 9.0 & 17.9 & 173.3 & 157 & 170.8: & 63.1 & $211.9^{*}$ & 216.2 & 1514 \\
\hline $15-16$ & 608 & $1.8:$ & 7.1 & 8.6 & 17.5 & 176.2 & 132 & 173.8: & 66.8 & 180.1 & 110.3 & 1412 \\
\hline $17-18$ & 745 & $3.2:$ & 9.5 & 7.5 & 14.7 & 177.6 & 98 & 141.1" : & 88.0 & 119.2 & 138.2 & 678.1 \\
\hline $19-20$ & 956 & $2.1 \%$ & 9.5 & 5.7 & 12.1 & 167.2 & 125 & 78.9: & 56.4 & 91.7 & 90.7 & 542.9 \\
\hline $21-22$ & 1804 & $4.8:$ & 10.5 & 8.9 & 16.9 & 195.8 & 181 & 72.5: & 56.7 & 88.2 & 99.3 & 428.7 \\
\hline Total & 6141 & 3.3 & 10.1 & 8.8 & 15.8 & 195.8 & 1009 & 127.2 & 98.5 & 156.5 & 144.8 & 1514 \\
\hline
\end{tabular}

$N$ - number of patients; effective dose - dose derived from dosimetric measurements on phantoms with scaling factors applied for age and exposure protocol; cumulative dose- represents the total individual dose provided by conventional x-rays and CBCT considering one year lag; $Q_{2}$-median effective dose, IQR - interquartile range; $M a x$ - maximum value of radiation dose, $\mu S v$ - microsievert; Relative Radiation Level ${ }^{23}$ and symbols were applied to effective dose as follow: $:$ for doses $<30 \mu \mathrm{Sv}$ in children under 18 year old and doses $<100 \mu \mathrm{Sv}$ for adults; $4 \%$ for doses of children aged under 18 years that ranged between 30 and $300 \mu \mathrm{Sv}$; * Mann Whitney $U$ test $\mathrm{p}<0.05$.

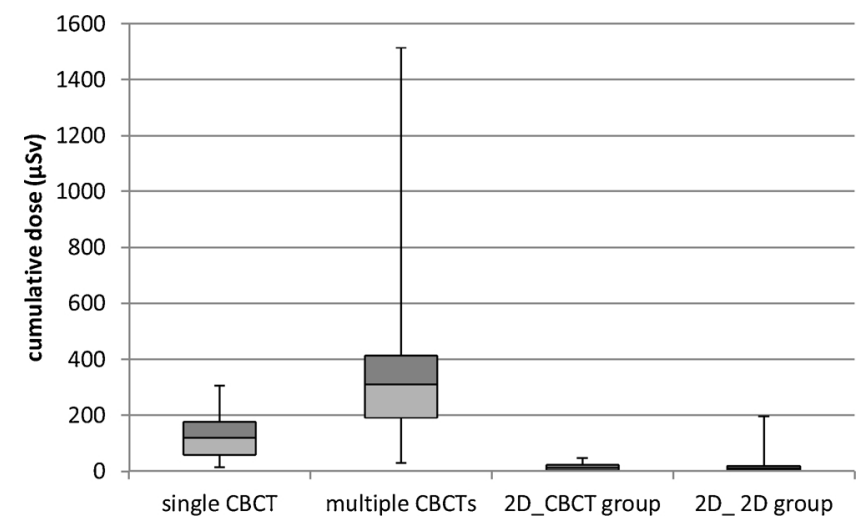

Fig. 1. Whisker-box-plots displays the distribution of the individual cumulative dose for dental radiological procedures in pediatric population: single CBCT: dose for CBCT incurred by patients exposed to a single CBCT examination; repeated $C B C T$ - dose for $\mathrm{CBCT}$ incurred by patients with multiple CBCTs within two years; $2 D_{-} C B C T$ group - individual dose accumulated by a patient from CBCT group from conventional dental radiological exposures; $2 D_{-} 2 D$ group individual dose accumulated by a patient from $2 \mathrm{D}$ group within two years.

and 15 years $(\mathrm{p}<0.0001)$ (Table 3$)$.

The overall contribution of the CBCT examinations to the collective dose was $70 \%$ in two years even though the number of CBCT exposures was lower than other dental radiological examinations.

\subsection{Radiation risk for pediatric cohort}

A higher Life Attributable Risk (LAR) was found in the CBCT group compared to 2D group of pediatric population, for all age intervals. The overall variation of LAR showed an increased risk for children exposed to CBCT at ages under 15 years $(\mathrm{p}<0.05)$ and the highest individual LAR was found for girls at the ages of 11-15 years (Table 3).

The background equivalent radiation time (BERT) for patients exposed to CBCT was higher than in the $2 \mathrm{D}$ group. The amount of radiation brought by a single СBCT examination was equivalent to 14.5 days of natural radiation on average and to 42.1 days for multiple CBCTs. The BERT for CBCT examination in the pediatric cohort was variable with age and the highest exposure equivalent time was found for ages between 11 and 15 years. The pediatric patients exposed only to conventional dental radiological examinations have a lower exposure equivalent time than for CBCT (1.5 days of natural radiation on average) which does not vary significantly with age (Table 3 ).

\section{Discussion}

\subsection{Radiation doses for dental radiological exposures}

An epidemiological study on CBCT radiation dose is challenging due to the heterogeneity of radiation exposure related to the radiological equipment, multiple combinations of exposure parameters, indications and patient characteristics. Currently, there are over 20 manufacturers providing more than 50 different CBCT units with different geometries

Table 3

Collective effective dose $(\mu \mathrm{Sv})$ and radiation risk for the studied groups.

\begin{tabular}{|c|c|c|c|c|c|c|c|c|c|c|}
\hline \multirow{3}{*}{$\begin{array}{l}\text { Age group } \\
\text { (years) }\end{array}$} & \multicolumn{3}{|c|}{ Per-caput collective dose ( $\mu$ Sv) } & \multicolumn{4}{|c|}{ Life attributable risk (LAR) of cancer incidence } & \multicolumn{3}{|c|}{ Background equivalent radiation time (BERT) (days) } \\
\hline & \multirow[t]{2}{*}{ 2D group } & \multirow[t]{2}{*}{ СBCT group } & \multirow[t]{2}{*}{ CBCT Contribution (\%) } & \multicolumn{2}{|c|}{$2 \mathrm{D}$ group } & \multicolumn{2}{|c|}{ CBCT group } & \multirow[t]{2}{*}{ 2D group } & \multicolumn{2}{|l|}{ СВСТ group } \\
\hline & & & & Female & Male & Female & Male & & Single CBCT & Multiple CBCT \\
\hline$<6$ & 11.2 & 131.8 & 71 & 0.4 & 0.2 & 6.3 & 1.9 & 1.3 & 12.8 & NA \\
\hline $6-10$ & 13.8 & 145.1 & 59 & 0.4 & 0.2 & 5.1 & 2.8 & 1.6 & 12.6 & 30.5 \\
\hline $11-15$ & 14.5 & $279.8^{*}$ & 84 & 0.3 & 0.1 & 7.1 & 2.8 & 1.7 & 20.7 & 55.1 \\
\hline $16-20$ & 11.5 & 159.1 & 64 & 0.1 & 0.1 & 3.8 & 1.7 & 1.4 & 11.5 & 35.8 \\
\hline $21-22$ & 13.1 & 108.7 & 44 & 0.2 & 0.1 & 2.8 & 1.1 & 1.5 & 9.2 & 22.2 \\
\hline Overall & 12.6 & 184.1 & $70 \%$ & 0.4 & 0.2 & 6.1 & 2.9 & 1.4 & 14.5 & 42.1 \\
\hline
\end{tabular}

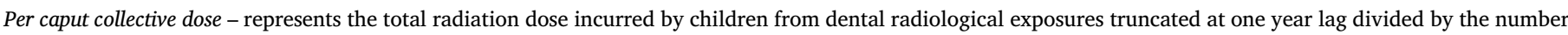

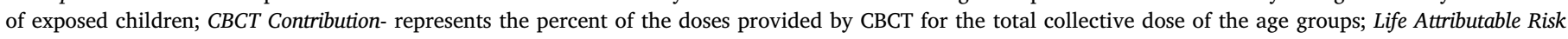

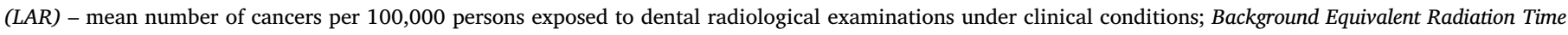

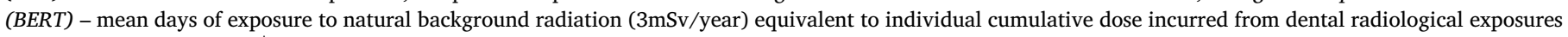
truncated at one year lag; "Mann Whitney $U$ test $\mathrm{p}<0.05 ; N A-$ not applicable. 
for scanning, collimators, filters and a large range of radiation dose per scan [24]. Moreover, most of these CBCT machines offer various protocols for exposures which considerably affect the radiation exposure including the FOV, milliampere $\mathrm{x}$ seconds (mAs), and kilovoltage $(\mathrm{kV})$, which can be controlled by the technician according to patient size and the image quality requirements.

Therefore, any estimation of CBCT irradiation on a large population expressed in terms of effective dose could provide only average approximations. Limitations of the effective dose metric for medical exposures, i.e. uncertainty of measurement methodology, discrepancy between tissue weighting factors and actual radiation-induced detriment, as well as gender- and age-averaging of tissue weighting factors, all apply to the current study; however, the effective dose can still be considered as the most suitable index for risk assessment at a population level, especially when comparing between imaging modalities. At the time of writing, the debate regarding the most suitable dose index for CBCT, and its conversion to patient dose, it still on-going. Currently available dose indices for CBCT (e.g. dose-area product) serve many purposes, but cannot be used to directly compare the radiation risk between different units or protocols. Instead, the results were derived from direct measurements of effective dose in literature, revealing differences between the patients who underwent a CBCT examination and those irradiated only with conventional dental radiological techniques and highlighted the amount of cumulative doses for a single and multiple CBCTs in the pediatric population (Fig. 1).

To the best of our knowledge, our study is the first survey that compares doses for children and young adults exposed to CBCT and exposed only to $2 \mathrm{D}$ dental radiological modalities, in terms of current dental practice and taking age into account. The results clearly showed that CBCT exposures increased the individual cumulative dose for dental radiological exposures in children. However, the median effective dose for CBCT was lower than $0.3 \mathrm{mSv}$ which placed this examination in the range of doses for mammography or pelvis radiography [23]. Also, the CBCT effective doses were much lower than the mean effective dose observed in children exposed to CT (1.06 mSv) [25].

On the other hand, a wide variation of the individual cumulative doses among ages was noted for children included in the CBCT group in contrast with the cumulative doses in the $2 \mathrm{D}$ group that do not vary significantly with age. The individual cumulative dose was significantly higher for children exposed to CBCT aged between 11 and 14 years compared to other ages (Mann Whitney $U$ test $\mathrm{p}<0.05$ ). One explanation could be the high variability of the exposure protocols and frequency of CBCT examinations for children with dental anomalies or growth anomalies that may require repeated CBCTs. The CBCT examination in cleft patients is used for teeth assessment during mixed and permanent dentition. Moreover, during this age period, the CBCT evaluation of the bone defect volume provides technical details for graft procedures and it is also useful for establishing the thickness of the cortical bone and the possibility or the limits of orthodontic teeth movements. The young adults with ages between 19 and 22 years were included in the pediatric cohort in order to cover the CBCT exposures used for orthognatic surgery planning. However, the present study was a retrospective one and justification for the dental radiological examinations was therefore not assessed.

Recently, the International Commission on Radiological Protection (ICRP) stated that the estimation of collective dose in a population will not give a good indication of the health consequences and risks for the patient populations, due to the differences of age distributions of the patients undergoing medical ionizing exposures [26]. Our study also confirmed the importance of age related reporting of doses for a child population considering the significant contribution of CBCT exposures to the collective dose among all ages.

European Commission of Radiological Protection recommends the separation of the collective doses into various components, with an estimation of doses for different groups, reflecting the age and exposure characteristics as type of examinations, number of examinations for the selected population in a certain period of time [27]. In light of these new trends, our results emphasized the differences of the cumulative dose between the CBCT and 2D group and the significantly increased dose in the repeated CBCT exposures.

\subsection{The radiation risk for pediatric cohort}

Children could have a potentially higher risk for radiation-induced cancer occurrence due to their longer life span and their higher radiosensitivity compared with adults [28]. So far, several risk models have been developed in order to estimate the risk of cancer incidence after low-dose exposures [29].

The risk estimations in the present study rely on the linear nonthreshold (LNT) model, which conservatively states that risks from high dose exposures can be linearly extrapolated to 0 , taking into account a dose and dose rate effectiveness factor (DDREF). Both the validity of the LNT model and the value of DDREF used for low doses have been under scrutiny, as it is based on epidemiological data with a high level of uncertainty.

The individual LAR for dental radiological exposures was significantly higher for the CBCT group of patients and the highest LAR was found for girls of 11-15 year old. However, it remains uncertain whether a causality association between the CBCT exposure and an increased risk of cancer incidence exists for children. Ongoing research on radiobiological effects of low doses will lead to a reconsideration of the LNT, and possible even a replacement with another dose-effect model, which could completely reshape the concept of radiation protection of medical exposures.

Another way of estimating the radiation risk for low dose exposure is to compare the medical radiological exposure with the background radiation that represents the level of radiation to which the entire population is exposed daily from natural radioactive substances ( $3 \mathrm{mSv}$ / year). The Background Equivalent Radiation Time (BERT) is a simple approach to express the potential risk for diagnostic radiological procedures. Our results showed that CBCT exposures increase the equivalent time of radiation compared to $2 \mathrm{D}$ group of patients but the equivalent risk remains much lower than for CT exposure $(0.5$ years for head CT) (22). BERT for CBCT was significantly higher for children exposed with multiple CBCTs between ages 11 and 15, being the age range for the highest dose per caput in the CBCT group (55.1 mean days equivalent time).

The American College of Radiology has established guidelines for defining the radiation dose, as a relative risk based on population medical exposure [23]. The Relative Radiation Level (RRL) classification allows for the comparison of the effective doses of medical exposures but the individual cumulative dose could be higher in children with multiple radiological examinations. The doses for 2D dental radiographies are equivalent with the radiation level for chest and hand radiographies while the CBCT examinations are placed in the range of doses for mammography and pelvis radiographies [23]. Nevertheless, in several cases with multiple CBCT exposures at ages under 16 years, the cumulative doses are much higher and can exceed $1 \mathrm{mSv}$. Further research is still needed to describe the potential biomolecular effects for low dose exposure.

\subsection{Limitation of the study}

The limitations of the present study are mainly related to the small size of the epidemiological cohort, estimation of CBCT doses for various ages of children, and the uncertainties of linear no-threshold model for cancer risk estimation in low dose exposures. Our study includes only a limited size of population, that gives a low statistical power in dose and risk estimations.

For this retrospective analysis of irradiation in dental radiological exposures, the dosimetric measurements reported in the literature were 
considered. Future prospective studies on a larger number of CBCTs and exposure protocols are still necessary to describe the radiation doses and risk for children exposed in clinical condition. A well-reasoned justification of CBCT examinations based on selection criteria could reduce the number of dental radiological exposures in children. Optimization protocols by minimizing the field of view (FOV) could improve the radiation safety in CBCT exposures [30].

The limitations of risk models for low dose and the presence of the baseline risk of cancer incidence makes it difficult to conclude that an increasing of doses in the group of children exposed to СBCT could be directly associated with an excess risk of cancer incidence. However, our results showed that doses for CBCT exposures in current clinical conditions are higher than for other dental radiological exposures, placing the СBCT examination in a higher relative radiation risk level that has been considered before for pediatric dentistry.

In conclusion, our results revealed a wide variability of doses and risks of CBCT exposures in young patients that should be considered for reporting the dose-risk model in dentistry. Dentists should weigh the benefits of exposure according to age and pathological condition when considering indicating a CBCT or a conventional 2D radiography.

\section{Authors contribution}

All authors had the same contribution as the first author.

\section{Conflict of interest statement}

All authors declared no financial or personal relationship with other people or organisation, that could influenced this work.

\section{Acknowledgements}

The research leading to these results has received funding from the "Open Project for the European Radiation Research Area" (OPERRA FP7 programme) under grant agreement no. 212246/2015-2017. The authors wish to thank to A. Coman from Department of Epidemiology of Polaris Medical Hospital for his contribution in the epidemiological research.

\section{Appendix A. Supplementary data}

Supplementary data associated with this article can be found, in the online version, at https://doi.org/10.1016/j.ejrad.2018.04.021.

\section{References}

[1] European Commission, Radiation Protection 180, Medical Radiation Exposure for the European Population, Office for Official Publications of the European Communities, Luxembourg, 2014Available from: https://ec.europa.eu/energy/en/ radiation-protection-publications.

[2] H.M. Alamri, M. Sadrameli, M.A. Alshalhoob, M. Sadrameli, M.A. Alshehri, Applications of CBCT in dental practice: a review of the literature, Gen. Dent. 60 (5) (2012) 390-400.

[3] European Commission, Radiation Protection 172. SEDENTEXCT Cone Beam CT for Dental and Maxillofacial Radiology: Evidence-based Guidelines, Office for Official Publications of the EuropeanCommunities, Luxembourg, 2012Available from: http://www.sedentexct.eu/files/radiation_protection_172.pdf.

[4] J.W. Casselman, K. Gieraerts, D. Volders, J. Delanote, K. Mermuys, B. De Foer, G. Swennen, Cone beam CT: non-dental applications, JBR-BTR 96 (6) (2013) 333-353.

[5] American Academy of Oral and Maxillofacial Radiology, Clinical recommendations regarding use of cone beam computed tomography in Orthodontics. Position statement by the American Academy of Oral and Maxillofacial Radiology, Oral. Surg. Oral Med. Oral Pathol. Oral Radiol. 116 (2) (2013) 238-257.

[6] R. Pauwels, J. Beinsberger, B. Collaert, C. Theodorakou, J. Rogers, A. Walker, L. Cockmartin, H. Bosmans, R. Jacobs, R. Bogaerts, K. Horner, SEDENTEXCT project consortium: effective dose range for dental cone beam computed tomography scanners, Eur. J. Radiol. 81 (2) (2012) 267-271.

[7] J.B. Ludlow, M. Ivanovic, Comparative dosimetry of dental CBCT devices and 64slice CT for oral and maxillofacial radiology, Oral Surg. Oral Med. Oral Pathol. Oral Radiol. Endod. 106 (2008) 106-114.

[8] A.C. Oenning, R. Jacobs, R. Pauwels, A. Stratis, M. Hedesiu, B. Salmon, Cone-beam CT in paediatric dentistry: DIMITRA project position statement, Pediatr. Radiol. (November) (2017).

[9] R. Pauwels, G. Zhang, C. Theodorakau, A. Walker, H. Bosman, R. Jacobs, K. Horner, R. Bogaerts, The SEDENTEXCT project consortium: effective radiation dose and eye lens dose in dental cone beam CT: effect of field of view and angle of rotation, Br. J. Radiol. 87 (2014) 20130654.

[10] J.A. Hidalgo-Rivas, C. Theodorakou, F. Carmichael, B. Murray, M. Payne, K. Horner, Use of cone beam CT in children and young people in three United Kingdom dental hospitals, Int. J. Paediatr. Dent. 24 (5) (2014) 336-348.

[11] J.B. Ludlow, R. Timothy, C. Walker, R. Hunter, E. Benavides, D.B. Samuelson, M.J. Scheske, Effective dose of dental CBCT-a meta analysis of published data and additional data for nine CBCT units, Dentomaxillofac. Radiol. 44 (1) (2015) 20140197.

[12] C.A. Raelson, K.M. Kanal, M.S. Vavilala, F.P. Rivara, L.J. Kim, B.K. Stewart, W.A. Cohen, Radiation dose and excess risk of cancer in children undergoing neuroangiography, AJR Am. J. Roentgenol. 193 (6) (2009) 1621-1628.

[13] R.T. Griffey, A. Sodickson, Cumulative radiation exposure and cancer risk estimates in emergency department patients undergoing repeat or multiple CT, AJR Am. J. Roentgenol. 192 (4) (2009) 887-892.

[14] B. Huang, J. Li, M.W. Law, J. Zhang, Y. Shen, P.L. Khong, Radiation dose and cancer risk in retrospectively and prospectively ECG-gated coronary angiography using 64. slice multidetector CT, Br. J. Radiol. 83 (986) (2010) 152-158.

[15] M. Loubele, R. Bogaerts, E. Van Dijck, R. Pauwels, S. Vanheusden, P. Suetens, G. Marchal, G. Sanderink, R. Jacobs, Comparison between effective radiation dose of CBCT and MSCT scanners for dentomaxillofacial applications, Eur. J. Radiol. 71 (3) (2009) 461-468.

[16] C. Theodorakou, A. Walker, K. Horner, R. Bogaerts, R. Jacobs, SEDENTEXCT project consortium: estimation of paediatric organ and effective doses from dental cone beam CT using anthropomorphic phantoms, Br. J. Radiol. 85 (1010) (2012) $153-160$.

[17] X.M. Qu, G. Li, J.B. Ludlow, Z.Y. Zhang, X.C. Ma, Effective radiation dose of ProMax 3D cone-beam computerized tomography scanner with different dental protocols, Oral Surg. Oral Med. Oral Pathol. Oral Radiol. Endod. 110 (2010) 770-776.

[18] J.B. Ludlow, L.E. Davies-Ludlow, S.C. White, Patient risk related to common dental radiographic examinations: the impact of 2007 International Commission on Radiological Protection recommendations regarding dose calculation, J. Am. Dent. Assoc. 139 (9) (2008) 1237-1243.

[19] F. Gijbels, R. Jacobs, R. Bogaerts, D. Debaveye, S. Verlinden, G. Sanderink, Dosimetry of digital panoramic imaging: part I: Patient exposure, Dentomaxillofac. Radiol. 34 (3) (2005) 145-149.

[20] A. Al-Okshi, A. M.Nilsson, M. Petersson, C. Wiese, Lindh using GafChromic film to estimate the effective dose from dental cone beam CT and panoramic radiography, Dentomaxillofac. Radiol. 42 (7) (2013) 20120343.

[21] National Research Council of the National Academies, Health Risks from Exposure to Low Levels of Ionizing Radiation: BEIR VII-Phase 2, The National Academy Press, 2006, Available from: https://www.nap.edu/read/11340/chapter/1.

[22] E.L. Nickoloff, Z.F. Lu, A.K. Dutta, J.C. So, Radiation dose descriptors: BERT, COD, DAP, and other strange creatures, Radiographics 28 (September-October (5)) (2008) 1439-1450.

[23] American College of Radiology ACR Appropriateness Criteria. Radiation Dose Assessment Introduction; 2011. Available at https://www.acr.org/-/media/ACR/ Files/Appropriateness-Criteria/RadiationDoseAssessmentIntro.pdf.

[24] R. Pauwels, Cone beam CT for dental and maxillofacial imaging; dose matters, Radiat. Prot. Dosimetry 165 (1-4) (2015) 156-161.

[25] J. Aw-Zoretic, D. Seth, G. Katzman, S. Sammet, Estimation of effective dose and lifetime attributable risk from multiple head CT scans in ventriculoperitoneal shunted children, Eur. J. Radiol. 83 (10) (2014) 1920-1924.

[26] International Commission on Radiological Protection The 2007 Recommendations of the International Commission on Radiological Protection. ICRP Publication 103, 2007 Ann ICRP 37 (2-4). Available from: http://www.icrp.org/publication.asp? id = ICRP $\% 20$ Publication $\% 20103$.

[27] European Commission on Radiological Protection Publication 154, 2008. European Guidance on Estimating Population Doses from Medical X- Ray Procedures. Available from: http://ddmed.eu/_media/background_of_ddm1:rp154.pdf.

[28] K.R. Kutanzi, A. Lumen, I. Koturbash, I.R. Miousse, Pediatric exposures to ionizing radiation: carcinogenic considerations, Int. J. Environ. Res. Public Health 13 (11) (2016) pii: E1057.

[29] M.P. Little, R. Wakeford, E.J. Tawn, S.D. Bouffler, A. Berrington de Gonzalez, Risks associated with low doses and low dose rates of ionizing radiation: why linearity may be (almost) the best we can do, Radiology 251 (1) (2009) 6-12.

[30] M.M. Rehani, R. Gupta, S. Bartling, G.C. Sharp, R. Pauwels, T. Berris, J.M. Boone, Radiological Protection in Cone Beam Computed Tomography (CBCT). ICRP Publication 129, Ann. ICRP 44 (1) (2015) Available from: http://www.icrp.org/ publication.asp?id = ICRP $\% 20$ Publication $\% 20129$. 